Diagnosis was dichotomized into osteoarthritis $(\mathrm{OA})$ and other diagnoses. Annual revision risks were calculated for each subsequent year after primary arthroplasty by dividing the number of revisions by the total number of patients at risk during that year. The risks were stratified according to the underlying diagnosis, sex, age at primary arthroplasty and fixation type. In addition cumulative annual revision percentages were calculated for the full follow-up period. Furthermore we estimated the percentage of avoided $\mathrm{OA}$ revisions by assuming that all $\mathrm{OA}$ patients received their primary THA 5 years later (in all age groups $<85 \mathrm{yrs}$ ) and that the revision risks remained the same in all age categories.

Results: In total 134463 primary THA patients were included of whom $68 \%$ were female, $89 \%$ had OA as underlying indication and $66 \%$ of the THAs were uncemented. The 7th year cumulative risk percentage varied between 2.0 and $11.7 \%$ (Table 1). Overall cumulative revision percentages were higher in younger age categories (Table 1), with the exception of a $11.7 \%$ revision in the group aged $85-90$ yrs (uncemented, male, other diagnosis), but this finding is likely due to chance as this group existed of 67 patients. We estimated that by delaying THA for 5 years, a total of 197 revision surgeries ( $4.4 \%$ of all revision surgeries) could be avoided, $48(14.0 \%)$ in the OA male cemented group, $11(0.9 \%)$ in the OA male uncemented group, $69(3.3 \%)$ in the OA female cemented group and $69(8.6 \%)$ in the female uncemented group. This could result in a yearly cost reduction of approximately 4 million euros.

Table 1. Cumulative revision percentages within 7 years after index surgery.

\begin{tabular}{|c|c|c|c|c|c|c|c|c|}
\hline \multirow[b]{3}{*}{$\begin{array}{l}\text { Age- } \\
\text { categories }\end{array}$} & \multicolumn{4}{|c|}{ Osteoarthritis } & \multicolumn{4}{|c|}{ Other diagnosis } \\
\hline & \multicolumn{2}{|c|}{ Male } & \multicolumn{2}{|c|}{ Female } & \multicolumn{2}{|c|}{ Male } & \multicolumn{2}{|c|}{ Female } \\
\hline & Cemented & | Uncemented & Cemented & Uncemented & Cemented & Uncemented & Cemented & Uncemented \\
\hline $50-54$ & 4,10 & 4,46 & 3,14 & 5,27 & 8,31 & 6,56 & 4,44 & 7,19 \\
\hline $55-59$ & 4,55 & 5,46 & 6,70 & 4,63 & 8,94 & 5,38 & 8,02 & 5,95 \\
\hline $60-64$ & 4,72 & 4,34 & 3,55 & 4,24 & 5,03 & 5,54 & 7,23 & 3,82 \\
\hline $65-69$ & 4,23 & 4,06 & 2,36 & 3,63 & 5,92 & 7,30 & 6,86 & 4,99 \\
\hline $70-74$ & 3.71 & 4,53 & 2,92 & 4,50 & 5,24 & 7,34 & 2,46 & 4,28 \\
\hline $75-79$ & 3,00 & 4,67 & 2,47 & 3,78 & 4,15 & 7,25 & 3,87 & 2,94 \\
\hline $80-84$ & 2,69 & 3,84 & 2,20 & 3,59 & 5,94 & 3,76 & 3,48 & 3,79 \\
\hline $85-90$ & 2,02 & 4,93 & 2,11 & 3,73 & 2,04 & 11,65 & 2,47 & 2,51 \\
\hline Total & 3,45 & 4,50 & 2,62 & 4,11 & 5,53 & 6,39 & 4,31 & 4,54 \\
\hline
\end{tabular}

Conclusions: Cumulative 7th year risk percentages decreased by age in all different categories. By delaying the primary THA surgery, revisions might be avoided thereby resulting in cost reduction.

Disclosure of Interest: None declared

DOI: 10.1136/annrheumdis-2017-eular.4274

\section{SAT0702 LOCAL AND SYSTEMIC INFLAMMATION IN PATIENTS WITH EARLY RHEUMATOID ARTHRITIS WITH CHLAMYDIA TRACHOMATIS INFECTION}

M. Natalia, K. Svetlana. Belorussian State Medical University, minsk, Belarus

Objectives: We had to study local and systemic inflammation in rheumatoid arthritis patients with persistence Chlamydia trachomatis (Ch tr) in the joint Methods: 31 patients with early RA; mean age $54,5(10,6)$ years, disease duration 21,5 $(14,4)$ weeks witch persistence $\mathrm{Ch}$ tr in the joint (mRNP $\mathrm{Ch}$ tr had been revealed in synovial fluid by NASBA PCR) were enrolled in this study. The comarison group was patients with RA $(n=42)$ without $m R N P$ in synovial fluid (Ch tr-). Mean age was 51,7 (15,4) years, disease duration 20,8 $(13,3)$ weeks. All the patients had been received only symptomatic treatment (NSAID). Disease activity had been detected by DAS 28 . Systemic inflammation was estimated by levels of erythrocyte sedimentation rate (ESR), hsp C-reactive protein (hspCRP), orozomucoid (OR) in the blood samples; local inflammation- by detection hsp CRP, OR in synovial fluid. Also we had been detected level of ACCP in the blood samples and synovial fluid.

Results: We didn't reveal statistically significant differences between levels of ESR, hsp CPR, OR, ACCP in blood samples patients with RA Chtr+ and RA Ch tr-. Level of hsp CPR and OR in synovial fluid of research group (Ch tr+) were significantly higher than comparison group $(4,1 \pm 0,3 \mathrm{mg} / \mathrm{l}$ versus $2,4 \pm 0,2 \mathrm{mg} / \mathrm{l}$, $\mathrm{p}<0,05$-hspCRP and 157,4 $\pm 17,5 \mathrm{mg} / \mathrm{dl}$ versus $78,5 \pm 18,9 \mathrm{mg} / \mathrm{dl}, \mathrm{p}<0,001-\mathrm{OR})$. In the group of research (Ch tr+) level of ACCP in synovial fluid was statistically significant higher than comparison group (Ch.tr -) $(195,6 \pm 37,3$ versus $67,9 \pm 15,4$; $\mathrm{p}<0,001)$

Conclusions: Patients with early RA detected by NASBA PCR in synovial fluid $\mathrm{Ch} \mathrm{tr}_{+}$, had been characterized by absence differences compared RA Ch trpatients in the level of systemic inflammation and had differences in the level of local inflammation. We revealed high level of ACCP in RA patients with $\mathrm{Ch}$ tr in the joints, that may be important for understanding some aspects of RA pathogenesis.

Disclosure of Interest: None declared

DOI: 10.1136/annrheumdis-2017-eular.6876

\section{SAT0703 INFLUENCE OF PHYSICAL ACTIVITY AND SLEEP ON FUNCTIONAL CAPACITY AND PAIN IN PATIENTS WITH KNEE OSTEOARTHRITIS}

M. Nuñez ${ }^{1}$, E. Nuñez ${ }^{2}$, J.M. Segur ${ }^{3}$, L. Lozano ${ }^{3}$, J. Montañana ${ }^{3}$, V. Segura ${ }^{3}$ M. Marti ${ }^{4}$, A. Garcia-Cardo ${ }^{4}$, S. Sastre ${ }^{3}$, X. Alemany ${ }^{3} .{ }^{1}$ Rheumatology and IDIBAPS Area 1, Hospital Clinic, ${ }^{2}$ SAP Suport al Diagnòstic $i$ al Tractament,
Institut Català de la Salut; ${ }^{3}$ Orthopedic Surgery and IDIBAPS; ${ }^{4}$ Rheumatology, Hospital Clinic, Barcelona, Spain

Background: Knee osteoarthritis $(O A)$ is a degenerative disease in which pain and functional disability progression tend to increase with reducing the healthrelated quality of life (HRQOL). Factors related to healthy lifestyles, such as physical activity and sleep, are known to have restorative benefits on function and pain in these patients. A previous study found that patients with reparative sleep achieved better WOMAC and SF-36 HRQOL questionnaire dimension scores.

Objectives: To determine the influence of physical activity and sleep on functional capacity and pain in patients with long-term knee OA.

Methods: Cross-sectional study. Sociodemographic and clinical variables, physical activity (PA) (regular physical exercise $\geq 3$ times a week $\geq 30$ minutes per session (PE) and sitting $\leq 6$ hours/day [S]) and sleep quality/reparative sleep (RS) were determined using the question: How do you usually sleep? (1=well [RS], $2=$ regular, $3=$ badly, $4=$ with medication/treatment [NRS]). Functional capacity and pain were evaluated using the WOMAC (specific) and SF-36 (generic) HRQOL questionnaires. Associations were analysed using multiple regression models. Results: 453 patients (84.3\% female), mean age 69.73 (8.4), BMI 35.27 [SD 6.3], comorbidities 2.43 (SD 1.5), 78.6\% with obesity (BMI 33.68 [SD 6.7]), depression/anxiety in $36.4 \%$, PE $60.5 \%$, S $72.2 \%$ and PA $48.6 \%$, were included. $22.5 \%$ reported RS. Bivariate analysis showed patients with PA and those with RS had better functional capacity and less pain intensity $(>10, p>0.001$, in both WOMAC and SF-36). The four multiple regression showed that patients with PA and SR had better scores, both in functional capacity (dependent variables, WOMAC and SF-36) and pain (dependent variables, WOMAC and SF-36), $p<0.006$. Age, gender, number of comorbidities and obesity were included in the models as potential confounders. Obesity was associated with worse function and more pain in the four models $(p<0.05)$. Being female and greater comorbidity were associated with poorer functional capacity and pain assessed by the SF-36. Conclusions: Physical activity and sleep were associated with less pain and better functional capacity, suggesting these variables should be determined systematically in clinical practice due to their significant relationship with HRQOL. Obesity was negatively associated with function and pain. There was also a negative relationship between female gender and comorbidity according to the SF-36. Differences in generic and specific questionnaires mean they should be used together to provide more detailed information.

Acknowledgements: This work was funded by project $\mathrm{Pl} / 13 / 00948$, integrated in the Plan Nacional I+D+I and cofounded by ISCIIISubdirección General de Evaluación and European Regional Development Fund (ERDF).

References:

[1] Uthman OA, van der Windt DA, Jordan JL, Dziedzic KS, Healey EL, Peat GM, et al. Exercise for lower limb osteoarthritis: systematic review incorporating trial sequential analysis and network meta-analysis. BMJ. 2013;347:f5555.

[2] Abad VC, Sarinas PS, Guilleminault C. Sleep and rheumatologic disorders. Sleep Med Rev 2008;12:211-28.

Disclosure of Interest: None declared

DOI: 10.1136/annrheumdis-2017-eular.2180

\section{SAT0704 THE IMPACT OF AUTOIMMUNE DISEASE IN THE MANAGEMENT AND PROGNOSIS OF ACUTE CORONARY SYNDROME}

${ }_{\text {N. Lozano }}{ }^{1}$, F.J. Pastor Perez ${ }^{2}$, L.F. LInares Ferrando ${ }^{1}$, J. Martinez Ferrin ${ }^{1}$ J. Ruiz Sara ${ }^{1}$, A. Paños Iniesta ${ }^{1}$, P. Castillo Dayer ${ }^{1}$, A. Bermudez Torrente ${ }^{1}$, F.A. Marinez Angosto ${ }^{1}$, S. Manzano Fernandez ${ }^{2} .{ }^{1}$ Rheumatology; ${ }^{2}$ Cardiology, University Hospital Virgen de la Arrixaca, Murcia, Spain

Background: Patients with autoimmune diseases (AID) have a high burden of cardiovascular disease leading to premature morbidity and mortality. But it is unclear if it is due to a higher prevalence of cardiovascular disease, to a worse case fatality or to a different management after an index event.

Objectives: The primary aim of the study is to assess the prognostic implications of the presence of AID both during the hospitalization and after discharge after an acute coronary syndrome (ACS). The secondary objectives included the assessment of the prevalence of AID in patients with ACS, their clinical profile and the management of this index event

Methods: The study included consecutive patients admitted after ACS from January 2011 to December 2015 at the University Hospital Virgen de la Arrixaca, Murcia (Spain). For AID patients, in-hospital management and ACS presentation was compared to non-AID patients. We also compared in-hospital and major adverse events during follow-up (death, recurrent non-fatal myocardial infarction, stroke and major bleeding, between groups). A multivariate Cox regression model was performed to assess the independent role of the presence of AID in the occurrence of the events of interest.

Results: Of 2236 patients included with ACS, 78 had AID (3.3\%): 24 rheumatoid arthritis, 10 inflammatory bowel disease, 7 ankylosing spondylitis, 6 psoriatic arthritis, 5 polymyalgia rheumatica, 2 systemic lupus erythematosus and 20 miscellanea. Mean age of AID patients was $67 \pm 13$ years and median evolution of the disease was 10 [4-14] years. Seventy percent of AID patients were taking corticosteroids, $50 \%$ disease modifying antirheumatic drugs, $22 \%$ non-steroidal anti-inflammatory drugs and 8 biological theraphy. No significant differences were found in clinical and demographics characteristics between groups except for a 
higher percentage of atrial fibrillation and chronic obstructive pulmonary disease in AID patients. Compared to non-AID patients, AID patients had similar clinical ACS presentation and no differences were found with respect to revascularization strategies or medical treatment at discharge. With respect to prognosis the two groups had comparable rates of adverse events during hospitalization ( $10 \%$ vs $10 \%, p=0.920$ ) with no statistically significant differences in any single event studied. However after a follow-up of 397 [375-559] years, AID patients had higher rate of combined adverse events $(44 \%$ vs $28 \% p<0,001)$. After multivariate adjustment the presence of AID was associated with increased total mortality (hazard ratio $2.1,95 \% \mathrm{Cl} 1.2$ to $3.7, \mathrm{p}=0.008$ ) and it was also a borderline risk factor for higher bleeding complications (hazard ratio $2.2,95 \% \mathrm{Cl} 0.9$ to 5.5 ). The presence of AID was not an independent risk factor for neither stroke or recurrent non-fatal myocardial infarction.

Conclusions: The presence of AID did not change ACS presentation and clinical management. Although AID is not associated with worse outcomes during hospitalization it is independently linked to higher total mortality and a trend to an increased risk of major bleeding during follow-up.

Disclosure of Interest: None declared

DOI: 10.1136/annrheumdis-2017-eular.6758

\section{SAT0705 DO DIET CHARACTERISTICS IMPACT EFFECT OF A 12-MONTH VITAMIN D SUPPLEMENTATION ON 25OH-VITAMIN D SERUM LEVEL AMONG OVERWEIGHT ELDERLY?}

R.M. Baddoura ${ }^{1}$, N.A. Yaghi ${ }^{2}$, A. Arabi ${ }^{3}$, G. Halaby ${ }^{4}$, G. El-Hajj-Fuleihan ${ }^{3}$. ${ }^{1}$ Rheumatology, Hotel-Dieu de France, St Joseph University; ${ }^{2}$ Nutrition, School of Pharmacy, St Joseph University; ${ }^{3}$ Calcium Metabolism, American University of Beirut Medical Center; ${ }^{4}$ Endocrinology, Hotel-Dieu de France, St Joseph University, Beirut, Lebanon

Background: Achieving desirable 250 Hvitamin $D$ serum levels with vitamin D supplementation may be influenced by many confounders including lifestyle patterns and vitamin D receptor genetic polymorphisms.

Objectives: To test the effect of dietary intake confounding variables such total as calories, dietary intake of vitamin $\mathrm{D}$, calcium, proteins, carbohydrates and fats, on the variance in serum 25-hydroxy vitamin D (25OHD) level after vitamin D supplementation

Methods: Study population: All subjects from one out of three centers who participated in a randomized clinical trial comparing high (3750 IU/day) versus low (600 IU/day) dose of vitamin D supplementation in ambulatory overweight (BMI exceeding 25) elderly subjects (age $\geq 65$ years), with serum 25OHD level between 10 and $30 \mathrm{ng} / \mathrm{ml}$ at screening.

Outcome measure: $25 \mathrm{OH}$ D serum levels at 12 months of follow-up.

Confounders included gender, body mass index, baseline 25OHD serum level, genetic polymorphism of vitamin D receptor (Fok, Bsml and Taq) and dietary intake of vitamin D, calcium, proteins, carbohydrates, fats and total calories assessed by food frequency and $24 \mathrm{~h}$ recall questionnaires, administered a year after the termination of the trial.

Statistical analysis: ANOVA for repeated measures and regression using STATA version 13. Numbers expressed as mean (SD).

Results: We studied 117 Participants, 62 females and 55 males, age was 71 (5) years, of who 61 received 3,750 IU of vitamin D, and 55 received 600 IU/day. Vitamin D receptor genetic polymorphisms distribution was comparable in both arms. Daily vitamin D intake excluding provided supplements was 7.59 (1.21) $\mathrm{mcg}$ and $8.53(1.36) \mathrm{mcg}$ from the $24 \mathrm{~h}$ recall, and food frequency questionnaires, respectively ( $p$ was NS between the two arms). Calories, calcium, protein, carbohydrate and fat mean intake was also similar across both arms. The high-dose group achieved significantly higher $25 \mathrm{OH}$-vitamin $\mathrm{D}$ serum levels at twelve months $34.9(9.3) \mathrm{ng} / \mathrm{ml}$ compared to low-dose, $21.9(6.1) \mathrm{ng} / \mathrm{ml}$ $(p<0.0001)$. Difference between arms remained significant adjusting for gender, baseline BMI, Fok, Bsml and Taq genetic polymorphism, baseline serum vitamin $\mathrm{D}$ and dietary intake of vitamin D, calcium, calories, proteins, carbohydrates and fats. None of these dietary intake measures was found to have significant contribution to the model variance. Supplementation dose was the only significant predictor of 250 Hvitamin D serum levels achieved at 12 months.

Conclusions: Diet composition was not a confounder for the effect of high versus low dose vitamin D 12-month supplementation, on achieved 25OHD vitamin D serum levels.

Acknowledgements: St Joseph University, Hotel-Dieu de France hospital

American University of Beirut Medical Centre

Lebanese National Council for Scientific Research

Lebanese Ministry of Social Affairs

Disclosure of Interest: None declared

DOI: 10.1136/annrheumdis-2017-eular.2206

\section{SAT0706 END-STAGE ORGAN FAILURE IN SARCOIDOSIS: CHARACTERIZATION AND PREDICTIVE FACTORS IN 1082 PATIENTS}

R. Pérez-Alvarez ${ }^{1}$, B. Kostov ${ }^{2}$, A. González García ${ }^{3}$, R. Gómez De La Torre ${ }^{4}$, M. Lopez Dupla ${ }^{5}$, B. De Escalante ${ }^{6}$, A. Alguacil ${ }^{7}$, J. Chara ${ }^{8}$, J. Velilla ${ }^{9}$, J. Rascón ${ }^{10}$, J.S. Garcia Morillo ${ }^{11}$, C. Feijoo ${ }^{12}$, E. Fonseca ${ }^{13}$, M. Bonet ${ }^{14}$,
J.L. Callejas ${ }^{15}$, G. de la Red ${ }^{16}$, A.B. Madroñero Vuelta ${ }^{17}$, C. Soler ${ }^{18}$, E. Peral ${ }^{19}$ J.F. Gómez Cerezo ${ }^{20}$, G. Cruz ${ }^{21}$, P. Perez ${ }^{22}$, S. Rodríguez Fernández ${ }^{23}$, B. Pinilla ${ }^{24}$, A. Gato ${ }^{25}$, M. Akasbi ${ }^{26}$, A. Robles ${ }^{27}$, I. Ojeda ${ }^{28}$, M.J. Vives ${ }^{29}$, C. Morcillo ${ }^{30}$, M. Penadés ${ }^{31}$ M. De Vicente ${ }^{32}$, M. Ramos-Casals ${ }^{33}$,

L. Pallarés ${ }^{10}$, P. Brito-Zerón ${ }^{30,33}$ on behalf of the SARCOGEAS-SEMI Registry. ${ }^{1}$ Hosp Alvaro Cunqueiro, Vigo; ${ }^{2}$ IDIBAPS, Barcelona; ${ }^{3}$ Hosp Ramón y Cajal, Madrid; ${ }^{4}$ HUCA, Oviedo; ${ }^{5}$ Hosp Joan XXIII, Tarragona; ${ }^{6}$ Hosp Clínico, Zaragoza; ${ }^{7}$ Hosp Virgen de la Salud, Toledo; ${ }^{8}$ Hosp Josep Trueta, Girona; ${ }^{9}$ Hosp Miguel Servet, Zaragoza; ${ }^{10}$ Hosp Son Espases, Palma de Mallorca; ${ }^{11}$ Hosp Virgen del Rocio, Sevilla; ${ }^{12}$ Hosp Parc Taulí, Sabadell; ${ }^{13}$ Hosp de Cabueñes, Gijón;

${ }^{14}$ Althaia, Manresa; ${ }^{15}$ Hosp Clínico San Cecilio, Granada; ${ }^{16}$ Hosp Esperit Sant, Santa Coloma; ${ }^{17}$ Hosp San Jorge, Huesca; ${ }^{18}$ Hosp de Sta Caterina, Girona:

${ }^{19}$ Hosp Virgen Macarena, Sevilla; ${ }^{20}$ Hosp Infanta Sofía, Madrid; ${ }^{21}$ Hosp de Poniente, Almería; ${ }^{22}$ Hosp Puerta del Mar, Cádiz; ${ }^{23}$ Hosp da Barbanza, A Coruña; ${ }^{24}$ Hosp Gregorio Marañón, Madrid; ${ }^{25} \mathrm{CH}$, Albacete; ${ }^{26}$ Hosp Infanta Leonor; ${ }^{27}$ Hosp la Paz, Madrid; ${ }^{28}$ Hosp Valle del Guadiato, Córdoba; ${ }^{29}$ San Joan de Déu, San Boi; ${ }^{30}$ Hosp CIMA-Sanitas, Barcelona; ${ }^{31}$ Hosp de Manises, Valencia; ${ }^{32}$ Hosp Nuestra Señora del Prado, Talavera; ${ }^{33}$ Hosp Clínic, Barcelona, Spain

Objectives: To characterize the main features at presentation of sarcoidosis associated with the development of end-stage organ failure in a large multicenter cohort of patients from Southern Europe.

Methods: In January 2017, the Spanish National Registry of Sarcoidosis (SARCOGEAS-SEMI) included 1082 consecutive patients diagnosed with sarcoidosis according to the ATS/ERS/WASOG 1999 statement and extrathoracic involvement to the 2014 WASOG instrument. The development of end-stage organ failure was assessed at the last visit.

Results: The cohort consisted of $618(57 \%)$ women and $464(43 \%)$ men, with a mean age at diagnosis of $47 y$ rs. After a mean follow-up of 82 months, 90 (8\%) patients developed end-stage organ failure, including respiratory failure $(n=56)$, chronic renal failure $(n=13)$, cardiac failure/permanent cardiac device $(n=8)$ and liver cirrhosis $(n=3)$. The following baseline features were associated with end-stage organ failure in the univariate analysis: patients born in Spain $(p=0.008)$, a higher mean age at diagnosis $(p<0.001)$ and a radiological stage III/IV $(p<0.001)$ With respect to extrathoracic involvement, spleen $(p=0.015)$, renal $(p=0.001)$, cardiac $(p=0.028)$ and bone marrow $(p=0.003)$ involvements, hypercalcemia $(p=0.018)$ and use of corticosteroids $(p<0.001)$ were associated with end-stage organ failure, while patients with cutaneous sarcoidosis had a lower risk $(\mathrm{p}=0.029)$. Multivariate analysis identified age at diagnosis (OR 1.05), radiological stages III/IV (OR 3.12) and use of corticosteroids (OR 4.55) as independent variables associated with the development of end-stage organ failure.

Conclusions: Nearly $10 \%$ of patients with sarcoidosis developed end-stage organ failure. Respiratory failure represented two thirds of cases of sarcoidosis-related organ failure, followed by renal ( $15 \%$ of cases) and cardiac $(9 \%)$. Older patients, as well as those presenting with advanced radiological stages, had an enhanced risk of developing end-stage organ failure.

Disclosure of Interest: None declared

DOI: 10.1136/annrheumdis-2017-eular.3376

\section{SAT0707 EFFICACY AND SAFETY OF PROPHYLACTIC TREATMENT ON ACTIVIATION OF LATENT TUBERCULOSIS DURING GLUCOCORTICOID THERAPY IN PATIENTS WITH RHEUMATIC DISEASES}

R. Wu. Department of Rheumatology, the First Affiliated Hospital of Nanchang University, Nanchang, China

Background: More than $80 \%$ of cases of tuberculosis are the result of reactivated latent infection, and nearly all these cases could be prevented by the administration of a course of antibiotic treatment. Therefore, it is suggested screening and treatment are most beneficial for those patients taking immunosuppressive medications. Although the prevalence of latent tuberculosis infection in China was much higher than the United States on the basis of tuberculin skin testing, In China, so far, no study has been done about prophylactic treatment on prevention of tuberculosis activation during glucocorticoid therapy in patients with rheumatic diseases. Should it been recommended that all patients with rheumatic diseases be screened for the presence of active tuberculosis or LTBI before the use of long term glucocorticoid or immunosuppressive medications in China? It is reported only about $5 \%$ of immunocompetent persons with a positive test will have progression from latent infection to disease in their lifetime. and decisions about whether to treat latent tuberculosis should take into account the individual patient's risk for the development of active tuberculosis and the risks of therapy. Objectives: To evaluate the risk of reactivation of LTBI in patients with rheumatic diseases who were undergoing prednisone use and the efficacy and safety of prophylactic treatment on prevention of tuberculosis reactivation.

Methods: 1000 patients with rheumatic diseases who were treated with prednisone were enrolled since 2012. IGRA test (the T-SPOT.TB test) were performed for all subjects. 50 patients with a IGRA-positive were administrated with rifampin for 4 months. 2-years follow-up was conducted to evaluate the risk factors of reactivation of LTBI and the efficacy and safety of rifampin treatment on prevention of tuberculosis reactivation. 